The electron-paramagnetic resonance linewidth of double-exchange interaction systems

This article has been downloaded from IOPscience. Please scroll down to see the full text article.

2004 J. Phys.: Condens. Matter 166293

(http://iopscience.iop.org/0953-8984/16/34/026)

The Table of Contents and more related content is available

Download details:

IP Address: 140.112.113.225

The article was downloaded on 17/07/2009 at 07:41

Please note that terms and conditions apply. 


\title{
The electron-paramagnetic resonance linewidth of double-exchange interaction systems
}

\author{
C D Hu \\ Department of Physics, National Taiwan University, Taipei, Taiwan, Republic of China
}

Received 25 January 2004

Published 16 August 2004

Online at stacks.iop.org/JPhysCM/16/6293

doi:10.1088/0953-8984/16/34/026

\begin{abstract}
We studied the electron-paramagnetic resonance linewidth of colossal magnetoresistance manganites. Starting from the quantum Langevin equation, we derived the transverse susceptibility of the double-exchange interaction systems and identified the damping function to be the inverse of the relaxation time $T_{2}$. We argued that the anisotropic energy caused by the crystal field was the most probable relaxation mechanism. The spin correlation caused by the double-exchange interaction was examined with the Schwinger boson approach. Taking into account the constraints of Schwinger bosons, we found that the linewidth is approximately proportional to the temperature. At the low-temperature end (near the Curie temperature $T_{C}$ ), there is a minimum, below which the linewidth increases sharply. At the high-temperature limit, the linewidth is proportional to $\sqrt{T / T_{C}}$. Most importantly, the linewidth is a universal function of $T / T_{C}$.
\end{abstract}

\section{Introduction}

It was found 50 years ago that perovskite manganites $\left(\mathrm{La}_{1-x} A_{x} \mathrm{MnO}_{3}\right.$, where $A$ is the divalent element and $x$ the doping concentration) show many interesting phenomena [1]. In the past 15 years, there has been a renewed interest in this kind of material due to the discovery of colossal magnetoresistance (CMR) [2]. Later studies [3] revealed a complex phase diagram, e.g. ferromagnetic insulator, ferromagnetic metal, paramagnetic insulator and antiferromagnetic insulator and very rich physics, e.g. charge ordering, orbit ordering and the Jahn-Teller effect. The fundamental mechanism in manganites is the double-exchange (DE) interaction, which was first proposed by Zener [4] and later developed by Anderson and Kasegawa [5] and de Gennes [6]. However, lattice distortion, orbital degeneracies and superexchange (SE) interaction all play significant roles $[7,8]$. The interplay of these interactions make these systems even more complex and intriguing.

Among the studies on manganites, there is the electron-paramagnetic resonance (EPR) experiment [9-15], which is very helpful for understanding the magnetism-related interaction and spin correlation. Peak position and peak intensity provide information about the $g$-factor and constituents. From the linewidth, one can find clues to the coupling strength, 
spin-relaxation mechanism and susceptibility. Results of previous experiments showed that the linewidth of EPR spectra in the paramagnetic phase increases almost linearly with temperature [10-14]. The origin is not clear. It was suggested to be spin-lattice relaxation [12]. A more recent analysis of experimental data [13] proposed an expression, $C(T-\theta) / T$, for linewidth and achieved a good fit. However, there is obviously more work to be done. The most important task is a rigorous derivation of linewidth from the fundamental mechanism. This is the aim of the present study. First, we construct the relation between susceptibility and the relaxation time of spins using the Langevin equation. This is presented in section 2 . In section 3, the DE interaction Hamiltonian is reduced to a simpler form. We calculate the spincorrelation function and susceptibility in section 4 and present the results with some discussion in section 5 .

\section{Formalism of transverse susceptibility}

The EPR lineshape can be computed from the transverse susceptibility. Suppose that, in addition to a static magnetic field $H_{0}$ in the $z$-direction, one applied microwaves with magnetic field in the $x$-direction to the system. The real (imaginary) part of the transverse susceptibility is given by the magnetization in the $x(y)$-direction divided by the microwave magnetic field. By solving the Bloch equations [16], the transverse susceptibility is given by

$$
\chi(\omega)=-\frac{\Omega \chi_{0}}{2\left(\omega-\Omega+i / T_{2}\right)},
$$

where $\Omega=g \mu_{\mathrm{B}} H_{0}$ and $\chi_{0}=M_{z} / H_{0}$, is the static susceptibility. The linewidth is usually denoted as $1 / T_{2}$. There are many ways to calculate the transverse susceptibility and, hence, $1 / T_{2}$. In this paper, we adopt the method of the quantum Langevin equation [17]. Using this method, we were able to treat a microscopic mechanism systematically. We start with the linear response theory. The negative imaginary part of the transverse susceptibility is given by [18]

$$
\chi^{\prime \prime}(\omega)=\frac{\left(g \mu_{\mathrm{B}}\right)^{2}}{4 V} \operatorname{Re} \int_{0}^{\infty} \mathrm{e}^{\mathrm{i} \omega t}\left\langle\left[S_{-}, S_{+}(t)\right]\right\rangle \mathrm{d} t
$$

where $\mathbf{S}$ is the total spin of the system; $\langle x\rangle$ represents the thermal average of operator $x$ and $\hbar$ was set to unity. Without loss of generality, the above equation can be written as

$$
\chi^{\prime \prime}(\omega)=\frac{\left(g \mu_{\mathrm{B}}\right)^{2}}{4 V} \operatorname{Re} \sum_{l, m}\left(S_{-}\right)_{m l}\left(S_{+}(\omega)\right)_{l m}\left(\mathrm{e}^{-\beta E_{m}}-\mathrm{e}^{-\beta E_{l}}\right) .
$$

Equation (3) can be calculated with the Langevin equation of $S_{+}(t)$,

$$
\frac{\mathrm{d} S_{+}(t)}{\mathrm{d} t}=-\mathrm{i} \Omega S_{+}(t)-\int_{0}^{t} \Phi(t-s) S_{+}(s) \mathrm{d} s+R(t),
$$

where $R(t)$ is the random force $(\langle R(t)\rangle=0)$ and $\Phi(t)$ is the damping function. After LaplaceFourier transform, equation (4) has the form

$$
S_{+}(\omega)=\frac{\mathrm{i}\left[S_{+}(t=0)+r(\omega)\right]}{\omega-\Omega+\mathrm{i} \phi(\omega)}
$$


where $r(\omega)$ and $\phi(\omega)$ are the Laplace-Fourier transforms of $R(t)$ and $\Phi(t)$, respectively. Substituting equation (5) into equation (3), one can calculate $\chi^{\prime \prime}(\omega)$,

$$
\chi^{\prime \prime}(\omega)=\frac{\left(g \mu_{\mathrm{B}}\right)^{2}}{4 V}\left(1-\mathrm{e}^{-\beta \Omega}\right) \operatorname{Re} \frac{-\mathrm{i}\left\langle S_{+} S_{-}\right\rangle}{\omega-\Omega+\mathrm{i} \phi(\omega)} .
$$

By comparing with equation (1), we identified $\operatorname{Re} \phi(\omega)$ with $1 / T_{2}$.

$\Phi(t)$ is a force-force correlation function. (Interested readers are referred to the works of Kubo [17] and Mori [19]),

$$
\Phi(t)=\frac{k_{B} T}{\left\langle S_{+} S_{-}\right\rangle} \int_{0}^{\beta} \mathrm{d} \lambda\left\langle\mathrm{e}^{\lambda \mathcal{H}} \dot{S}_{+}(t) \mathrm{e}^{-\lambda \mathcal{H}} \dot{S}_{-}\right\rangle
$$

There are at least two ways of calculating $\phi(\omega)$. Mori [19] developed a method of continual fractions. We found that the fractions quickly become complicated and intractable in the double-exchange system. Therefore we adopted the approach proposed by Ting and Nee [20]. Inserting complete sets of states into equation (7), we obtain

$$
\Phi(t)=\frac{1}{\left\langle S_{+} S_{-}\right\rangle} \sum_{n, m} \frac{\mathrm{e}^{-\beta E_{m}}-\mathrm{e}^{-\beta E_{n}}}{\beta E_{n m}} \dot{S}_{+n m} \dot{S}_{-m n} \mathrm{e}^{\mathrm{i} E_{n m} t}
$$

Hence,

$$
\phi(\omega)=-\frac{1}{\left\langle S_{+} S_{-}\right\rangle} \sum_{n, m} \frac{\mathrm{e}^{-\beta E_{m}}-\mathrm{e}^{-\beta E_{n}}}{\beta E_{n m}} \frac{\dot{S}_{+n m} \dot{S}_{-m n}}{\mathrm{i} \omega+\mathrm{i} E_{n m}-\eta} .
$$

Its real part is

$$
\operatorname{Re} \phi(\omega)=\frac{\pi}{\left\langle S_{+} S_{-}\right\rangle} \sum_{n, m} \frac{\mathrm{e}^{-\beta E_{m}}-\mathrm{e}^{-\beta E_{n}}}{\beta E_{n m}} \dot{S}_{+n m} \dot{S}_{-m n} \delta\left(\omega+E_{n m}\right)
$$

Finally, we have

$$
\operatorname{Re} \phi(\omega)=\operatorname{Im} \frac{\psi(\omega)-\psi(0)}{\omega},
$$

where

$$
\psi(\omega)=\frac{\mathrm{i} k_{B} T}{\left\langle S_{+} S_{-}\right\rangle} \int_{0}^{\infty} \mathrm{d} t\left\langle\left[\dot{S}_{-}, \dot{S}_{+}(t)\right]\right\rangle \mathrm{e}^{\mathrm{i} \omega t}
$$

is the response function of $\dot{S}_{+}$. Equation (11) is readily obtained by inserting complete sets of states between the operators of equation (12). Now, we are ready to apply the above method to the physical systems we are interested in. Our strategy is to calculate the relevant terms of $\psi(\omega)$ with Matsubara formalism and then use analytical continuation. The result is substituted into equation (11) to evaluate the linewidth.

\section{Double-exchange interaction}

The fundamental mechanism in CMR manganites is the DE interaction. The Hamiltonian has the following form:

$$
H=-t \sum_{\mathbf{k}, \sigma} c_{\mathbf{i} \sigma}^{\dagger} c_{\mathbf{j} \sigma}+J \sum_{\mathbf{i}} \mathbf{S}_{\mathbf{i}} \cdot \sigma_{\mathbf{i}}+g \mu_{\mathrm{B}} H_{0} \sum_{\mathbf{i}} S_{\mathbf{i} z}
$$


The first term of equation (13) describes the hopping of the holes between $\mathrm{Mn}^{+3}$ and $\mathrm{Mn}^{+4}$ sites. The second term is Hund's coupling, when combined, they give the DE interaction. The third term is due to the applied field. In the hole picture, the manganese ions have a spin, $S=2$. Whenever a hole is present, the local spin is coupled strongly with the spin of the hole. According to Hund's rule, the antiparallel state has the lower energy. The level spacing $J$ is of the order of eV and, hence, is the largest energy in the system. To treat it correctly, we used the approach proposed by Kubo and Ohata [21]. They used projection operators to impose a restriction on holes. Hoppings can only occur at those sites where the local spins are antiparallel to those of holes. Thus, the first and second terms of equation (13) are replaced by

$$
H_{D E}=-t \sum_{\mathbf{i}, \mathbf{j}, \alpha, \beta, \gamma} c_{\mathbf{i} \alpha}^{\dagger} P_{\mathbf{i} \alpha \gamma} P_{\mathbf{j} \gamma \beta} c_{\mathbf{j} \beta},
$$

where $\alpha, \beta$ and $\gamma$ are spin indices and

$$
P_{\mathbf{i}}=\frac{S-\mathbf{S}_{\mathbf{i}} \cdot \sigma}{2 S+1}
$$

with $\sigma / 2$ denoting the spins of the conduction holes. It has been shown that $H_{D E}$ in equation (14) is equivalent to the following Hamiltonian [22-24]:

$$
H_{D E}=-\frac{t}{2 S+1} \sum_{\mathbf{i}, \mathbf{j}} f_{\mathbf{i}}^{\dagger}\left(A_{\mathbf{j}}^{\dagger} A_{\mathbf{i}}+B_{\mathbf{j}}^{\dagger} B_{\mathbf{i}}\right) f_{\mathbf{j}},
$$

where the slave-fermion method was applied:

$$
\begin{aligned}
& c_{i \uparrow}=f_{i} \alpha_{i}, \\
& c_{i \downarrow}=f_{i} \beta_{i}
\end{aligned}
$$

and the spin-up and spin-down operators $\alpha$ and $\beta$ combined with the localized spins were represented by the Schwinger boson operators $A$ and $B$ :

$$
\begin{aligned}
& A^{\dagger}|S-1 / 2, m-1 / 2\rangle=\sqrt{S+m}|S, m\rangle, \\
& B^{\dagger}|S-1 / 2, m+1 / 2\rangle=\sqrt{S-m}|S, m\rangle .
\end{aligned}
$$

There is a constraint due to the slave fermion and Schwinger boson approaches [25]

$$
A_{\mathbf{i}}^{\dagger} A_{\mathbf{i}}+B_{\mathbf{i}}^{\dagger} B_{\mathbf{i}}+f_{\mathbf{i}}^{\dagger} f_{\mathbf{i}}=2 S \text {. }
$$

In the momentum space, we have

$$
H_{D E}=-\frac{1}{N(2 s+1)} \sum_{\mathbf{k}, \mathbf{p}, \mathbf{q}} E_{\mathbf{k}-\mathbf{p}-\mathbf{q}} f_{\mathbf{k}-\mathbf{q}}^{\dagger}\left(A_{\mathbf{p}} A_{\mathbf{p}+\mathbf{q}}^{\dagger}+B_{\mathbf{p}} B_{\mathbf{p}+\mathbf{q}}^{\dagger}\right) f_{\mathbf{k}}
$$

where

$$
E_{\mathbf{k}}=t \sum_{n . n .} \mathrm{e}^{\mathrm{i} \mathbf{k} \cdot \mathbf{R}_{n n}}
$$

with the summation being over the nearest neighbours. It is clear that the bosons and fermions are closely related to each other. To show the implications of the Hamiltonian in equation (20), we give the boson and fermion energies at the mean-field level, respectively, as follows:

$$
\omega_{\mathbf{q}}=\frac{1}{N(2 s+1)} \sum_{\mathbf{k}} E_{\mathbf{k}+\mathbf{q}}\left\langle f_{\mathbf{k}}^{\dagger} f_{\mathbf{k}}\right\rangle
$$


and

$$
\varepsilon_{\mathbf{k}}=\frac{1}{N(2 s+1)} \sum_{\mathbf{p}} E_{\mathbf{k}+\mathbf{p}}\left\langle A_{\mathbf{p}}^{\dagger} A_{\mathbf{p}}+B_{\mathbf{p}}^{\dagger} B_{\mathbf{p}}\right\rangle .
$$

Thus we have simplified the DE Hamiltonian considerably.

There exists an SE interaction between manganese ions. The manganites exhibit an antiferromagnetic phase whenever the DE interaction is diminished. The Néel temperature is about $200 \mathrm{~K}$. This indicates that the SE interaction coupling $J_{S E}$ is of the order $1 \mathrm{meV}$ or $t / 100$. In the temperature range $300-1000 \mathrm{~K}$, the thermal fluctuation is more important than that caused by the SE interaction. The latter can be accounted for with random-phase approximation [26]. The interaction between manganese ions (indirect exchange through the DE interaction) is 'screened' by the factor, $1+J_{S E} /\left(i \omega_{n}-\omega_{0}\right)$, where $\omega_{0}$ is the spin-level splitting. This, in effect, decreases the spin stiffness and the $T_{C}$. In the following calculation, we more or less incorporate the effect of the SE interaction by using an effective spin stiffness. On the other hand, as the fluctuation caused by the SE interaction is more important near the $T_{C}$, we expect the slope of $1 / T_{2}$ to decrease as the low-temperature end is lifted.

\section{Relaxation}

The reason for broadening in the EPR spectra is not clear. The dipolar interaction is too small in magnitude. It produces a linewidth of a few G. The Dzyaloshinsky-Moriya (DM) interaction [27] is a possible candidate. The ESR data of Alejandro et al [28] on $\mathrm{La}_{7 / 8} \mathrm{Sr}_{1 / 8} \mathrm{MnO}_{3}$ suggests that the DM interaction is of the order $0.4 \mathrm{~K}$. The data of [29] gives the order of $1 \mathrm{~K}$, the analysis of Huber et al [30] gives $0.8 \mathrm{~K}$ and that of Deisenhofer et al [31] gives $0.25 \mathrm{~K}$. However, note that all the above studies are concerned with the insulating region, i.e. small doping. In the metallic region that we are interested in, the DM interaction will be much smaller for the following reasons. First, the lattice distortion is smaller. Secondly, the electron orbits are less localized and the orbital characteristics are less manifest. Lastly, the broadening should have been closely related to the Lande- $g$ factor if indeed the DM interaction was the cause [27]. Experimental data [13] showed that $g \simeq 2.0$, an indication that the DM interaction is not robust.

Lattice distortion is another possibility; however, the phonon-related mechanism should have given a different temperature dependence. We have a more detailed discussion in the next section. The remaining candidate is the anisotropy energy of spins. In manganites, a manganese ion and neighbouring oxygen atoms formed an octahedron. Two octahedrons were rotated in the opposite direction and the bond angle of $\mathrm{Mn}-\mathrm{O}-\mathrm{Mn}$ deviated from $\pi$. Incidentally, experiments [32] showed that the bond angle affected the bandwidth, hence, it is an important parameter. For our purpose, the deviation of bond angle or bond bending broke significantly the cubic symmetry. In a system of cubic symmetry, the degeneracy of d-orbitals was lifted partially to $\mathrm{e}_{\mathrm{g}}$ and $\mathrm{t}_{2 \mathrm{~g}}$ orbitals. At systems with still lower symmetry, such as those in the presence of tetragonal distortion, an additional ligand field should be introduced into the Hamiltonian. It takes the form $B_{2} O_{2}^{0}$, where $O_{2}^{0}$ is a spin operator and $B_{2}$ is the field strength [33]. Thus, we have the following general form of an additional term in our Hamiltonian:

$$
H_{a n i}=\sum_{\mathbf{i}}\left(F S_{\mathbf{i} x}^{2}+G S_{\mathbf{i} y}^{2}\right)
$$

where $F$ and $G$ are constants. Since the DE interaction conserves total spin, we found

$$
\begin{aligned}
\dot{S}_{+} & =\mathrm{i}\left[H_{a n i}, S_{+}\right] \\
& =-\frac{\mathrm{i}}{2} \sum_{\mathbf{i}}\left[(F-G)\left(S_{\mathbf{i}-} S_{\mathbf{i} z}+S_{\mathbf{i z}} S_{\mathbf{i}-}\right)+(F+G)\left(S_{\mathbf{i}+} S_{\mathbf{i} z}+S_{\mathbf{i} z} S_{\mathbf{i}+}\right)\right] .
\end{aligned}
$$


Hence,

$$
\begin{aligned}
\left\langle\left[\dot{S}_{+}(t), \dot{S}_{-}\right]\right\rangle= & \frac{1}{4} \sum_{\mathbf{i}, \mathbf{j}}\left\{(F-G)^{2}\left\langle\left[S_{\mathbf{i} z}(t) S_{\mathbf{i}-}(t)+S_{\mathbf{i} z}(t) S_{\mathbf{i}-}(t), S_{\mathbf{j} z} S_{\mathbf{j}+}+S_{\mathbf{j}+} S_{\mathbf{j} z}\right]\right\rangle\right. \\
& \left.+(F+G)^{2}\left\langle\left[S_{\mathbf{i} z}(t) S_{\mathbf{i}+}(t)+S_{\mathbf{i}+}(t) S_{\mathbf{i} z}(t), S_{\mathbf{j} z} S_{\mathbf{j}-}+S_{\mathbf{j}-} S_{\mathbf{j} z}\right]\right\rangle\right\} .
\end{aligned}
$$

Since the calculation was performed in the paramagnetic phase, $\mathbf{i}$ and $\mathbf{j}$ have to be equal. Furthermore, equation (26) can be simplified to the following form since the decouplings, such as $\left\langle S_{\mathbf{i} z}(t) S_{\mathbf{i}+}(t) S_{\mathbf{i} z} S_{\mathbf{i}-}\right\rangle \approx\left\langle S_{\mathbf{i} z}(t) S_{\mathbf{i} z}\right\rangle\left\langle S_{\mathbf{i}+}(t) S_{\mathbf{i}-}\right\rangle$, are good approximations in the paramagnetic phase:

$$
\left\langle\left[\dot{S}_{+}(t), \dot{S}_{-}\right]\right\rangle=\frac{\left\langle S_{z}^{2}\right\rangle}{N} \sum_{\mathbf{i}}\left\{(F-G)^{2}\left\langle\left[S_{\mathbf{i}_{-}}(t), S_{\mathbf{i}_{+}}\right]\right\rangle+(F+G)^{2}\left\langle\left[S_{\mathbf{i}_{+}}(t), S_{\mathbf{i}_{-}}\right]\right\rangle\right\} .
$$

To calculate $\psi(\omega)$, we replaced the spin operators by Schwinger boson operators

$$
\begin{aligned}
& S_{+\mathbf{i}}=A_{\mathbf{i}}^{\dagger} B_{\mathbf{i}}, \\
& S_{-\mathbf{i}}=B_{\mathbf{i}}^{\dagger} A_{\mathbf{i}},
\end{aligned}
$$

and introduced their respective Green functions

$$
\begin{aligned}
& \widetilde{D}_{i A}(\tau)=-\left\langle T A_{i}(\tau) A_{i}^{\dagger}\right\rangle, \\
& \widetilde{D}_{i B}(\tau)=-\left\langle T B_{i}(\tau) B_{i}^{\dagger}\right\rangle .
\end{aligned}
$$

Substituting equations (27)-(29) into equation (12), we obtained

$$
\begin{gathered}
\psi\left(\mathrm{i} \omega_{n}\right)=\frac{k_{B} T}{2 N} \sum_{\mathbf{i}} \int_{0}^{\beta} \mathrm{d} \tau \mathrm{e}^{\mathrm{i} \omega_{n} \tau}\left[(F-G)^{2} \widetilde{D}_{i A}(-\tau) \widetilde{D}_{i B}(\tau)\right. \\
\left.+(F+G)^{2} \widetilde{D}_{i A}(\tau) \widetilde{D}_{i B}(-\tau)\right]
\end{gathered}
$$

In the paramagnetic phase, the zeroth-order spin propagators are

$$
D_{A}\left(\mathbf{q}, \mathrm{i} \omega_{n}\right)=D_{B}\left(\mathbf{q}, \mathrm{i} \omega_{n}\right)=\frac{1}{\mathrm{i} \omega_{n}-\omega_{\mathbf{q}}} .
$$

Applying Fourier transform and Matsubara summation, we obtain

$$
\psi\left(\mathrm{i} \omega_{n}\right)=\frac{k_{B} T}{N^{2}}\left(F^{2}+G^{2}\right) \sum_{\mathbf{p}, \mathbf{q}} \frac{n_{\mathbf{p}}-n_{\mathbf{q}}}{\mathrm{i} \omega_{n}-\omega_{\mathbf{p}}+\omega_{\mathbf{q}}},
$$

where $n_{\mathbf{p}(\mathbf{q})}$ is the Bose-Einstein distribution function. Substituting equation (32) into equation (11), we obtained

$$
\operatorname{Re} \phi(\omega)=-\frac{\pi k_{B} T}{N^{2}}\left(F^{2}+G^{2}\right) \sum_{\mathbf{p}, \mathbf{q}} \frac{n_{\mathbf{q}}-n_{\mathbf{p}}}{\omega_{\mathbf{q}}-\omega_{\mathbf{p}}} \delta\left(\omega-\omega_{\mathbf{p}}+\omega_{\mathbf{q}}\right) .
$$

If one writes

$$
\omega_{\mathbf{q}}=D q^{2}
$$

where $D$ is the spin stiffness, equation (33) can be reduced to

$$
\begin{aligned}
\operatorname{Re} \phi(0) & =-\frac{\pi k_{B} T V^{2}}{(2 \pi)^{6} N^{2}} \int \mathrm{d}^{3} p \int \mathrm{d}^{3} q \delta\left(\omega_{\mathbf{p}}-\omega_{\mathbf{q}}\right) \frac{\partial n_{\mathbf{p}}}{\partial \omega_{\mathbf{p}}} \\
& =-\frac{\left(k_{B} T a^{3}\right)^{2}}{2(2 \pi D)^{3}}\left(F^{2}+G^{2}\right) \ln |1-z|,
\end{aligned}
$$


with $z$ being the fugacity and $a$ the lattice constant. The fugacity is related to the constraint of the Schwinger bosons. In view of equation (19), the constraint in momentum space is

$$
\sum_{\mathbf{q}} A_{\mathbf{q}}^{\dagger} A_{\mathbf{q}}=N(S-x / 2)
$$

where we have made the approximation of replacing local constraints with a global constraint and used the fact that in the paramagnetic phases $A$ and $B$, bosons are equally populated. It is important to distinguish between $D$ and the usual spin wave stiffness. The relation between spin operators and Schwinger bosons are, for example, $S_{+}=A^{\dagger} B$. Clearly, they are not the same. The spin wave propagator is a convolution of those of Schwinger bosons. Spin wave stiffness should be computed accordingly. Here, we are not concerned with spin waves. Equation (36) can be written as

$$
\left(\frac{k_{B} T a^{2}}{4 \pi D}\right)^{3 / 2} g_{3 / 2}(z)+\frac{z}{N(1-z)}=(S-x / 2),
$$

where the second term comes from the state at $q=0$ [34], and

$$
g_{n}(z)=\frac{1}{\Gamma(n)} \int_{0}^{\infty} \frac{x^{n-1} \mathrm{~d} x}{z^{-1} \mathrm{e}^{x}-1}
$$

with $\Gamma(n)$ being the gamma function. It is necessary for the ferromagnetic phase for the following reasons. If the dispersion relation of equation (22) were used, we would have $D \approx x t a^{2} /(2 S+1)$. For a typical system of $t=0.1 \mathrm{eV}, x=0.3$ and $T=200 \mathrm{~K}$, the prefactor of $g_{3 / 2}(z)$ is approximately 0.13 . Since $S=2$ and the largest value of $g_{3 / 2}(z)$ is $g_{3 / 2}(1) \approx 2.6$, the presence of the second term of equation (37) is necessary. This is an indication that the spin stiffness given above needs to be revised. It is valid at a low temperature. As the temperature increases, the motion of holes will be impeded by spin fluctuations. As a result, the coupling between spins will be weakened and the spin stiffness is decreased. The computation of the actual value of $D$ is very complicated and beyond the scope of this paper. Hence, we used a simple approach here. We assume that, at the Curie temperature, the second term on the left hand side of equation (37) vanishes, i.e.

$$
\left(\frac{k_{B} T_{C} a^{2}}{4 \pi D}\right)^{3 / 2} g_{3 / 2}(1)=(S-x / 2) .
$$

We evaluated $D$ in this way. Above $T_{C}$, the system is always insulating and the spin orientations are random. We can assume that $D$ remains more or less temperature-independent in the paramagnetic phase. Now, the fugacity can be computed with the following equation:

$$
\left(\frac{T}{T_{C}}\right)^{3 / 2} \frac{g_{3 / 2}(z)}{g_{3 / 2}(1)}=1
$$

for temperatures greater than $T_{C}$. Substituting equation (39) into (35), we obtained

$$
\frac{1}{T_{2}}=\operatorname{Re} \phi(0)=-4 \frac{F^{2}+G^{2}}{k_{B} T_{C}}\left(\frac{S-x / 2}{g_{3 / 2}(1)}\right)^{2}\left(\frac{T}{T_{C}}\right)^{2} \ln |1-z| .
$$

Equations (40) and (41) are our final result.

In retrospect, the major mathematical structure is the factor $\partial n_{\mathbf{p}} / \partial \omega_{\mathbf{p}}$ in equation (35). It is quite robust. Its presence is not affected by taking into account the self-energy of boson propagators. We show this in the appendix. Hence, the expression in equation (41) seems to be universal. 


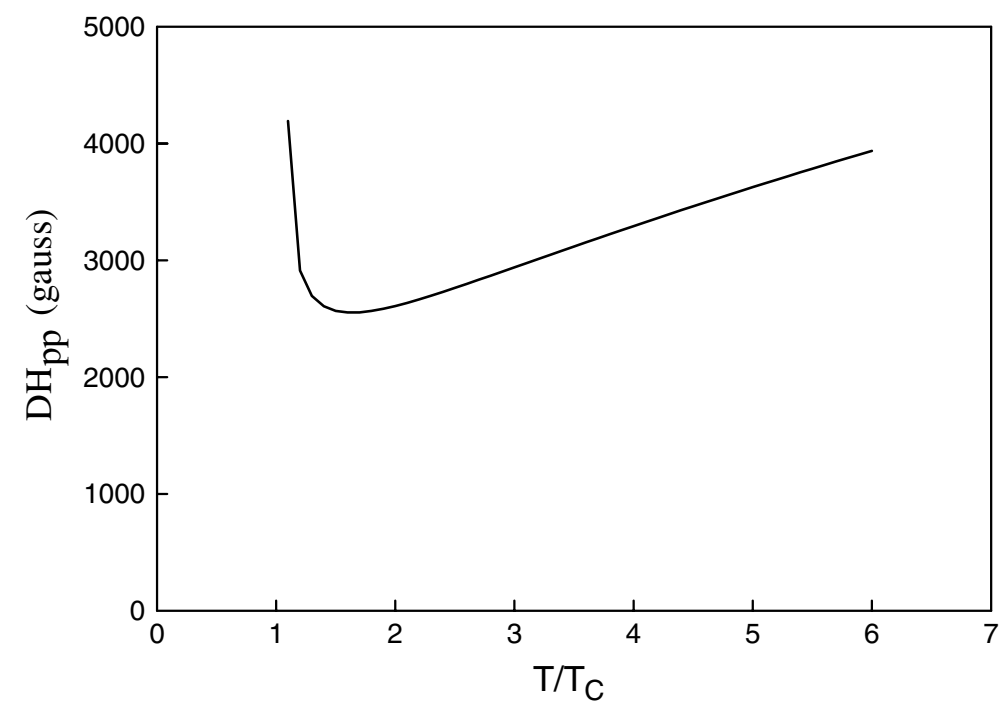

Figure 1. The linewidth, $\Delta H_{p p}$, or the peak-to-peak magnetic field difference against $T / T_{C}$ for $F=G=2 \mathrm{~K}$.

\section{Results and discussion}

All the experimental data showed that the EPR linewidth of manganites with doping between 0.2 and 0.4 has a quasi-linear dependence on temperature. Our calculation has a similar result (figure 1). At the low-temperature end (near Curie temperature $T_{C}$ ), there is a minimum. Below the minimum, the linewidth increases sharply. With increase in temperature, the linewidth increases almost linearly. It bends downward in the high-temperature region. The quasi-linear dependence on temperature has been attributed to various sources, notably the one-phonon spinlattice relaxation proposed by Huber and Seehra (HS) [35] and Dormann-Jaccarino-Huber's (DJH) [36-38] consideration of dipolar interaction or any other temperature-independent interaction. In any case, spin-lattice relaxation cannot give the correct temperature dependence. For example, in equation (A-5) of [3], $\chi_{T} k_{B} T$ is almost constant in the high-temperature region. The phonon distribution function $n_{\mathbf{p}, \mathbf{q}}$ gives a factor of $k_{B} T$. The spin correlation function introduces additional temperature dependence, except for, perhaps, in the high-temperature limit. Thus $1 / T_{2}$ cannot be quasi-linear. As for DJH's explanation, we found in Huber's original derivation $[37,38]$ that $1 / T_{2}$ is proportional to $k_{B} T \xi^{5 / 2}$. The correlation length $\xi$ has to be temperature-independent for $1 / T_{2}$ to be linearly proportional to $T$. This is unlikely and, hence, we concluded that neither can produce linear temperature dependence.

There is a sharp upturn at the low-temperature end. Experimentally, all the powder samples showed this kind of behaviour. Data from single-crystal samples are not conclusive. Therefore there was ambiguity as to whether it is a sample-dependent effect. Our calculation showed that it is intrinsic. The cause is the divergence of $\ln (1-z)$ at $T_{C}$. It is universal. At temperatures close to $T_{C}[39]$,

$$
g_{3 / 2}(z) \simeq g_{3 / 2}(1)-3.54 \sqrt{\delta z},
$$

where $\delta z=1-z$. In view of equation (40), we obtained

$$
\delta z \simeq 1.23 \varepsilon^{2},
$$


where $\varepsilon=T / T_{C}-1$. Thus we concluded that, near $T_{C}$, the divergence is of the form

$$
\frac{1}{T_{2}} \propto-\ln \varepsilon .
$$

The high-temperature behaviour of linewidth is straightforward. In view of equation (40), $g_{3 / 2}(z)$ and, hence, $z$ is small. Since

$$
g_{3 / 2}(z)=\sum_{n} \frac{z^{n}}{n^{3 / 2}}
$$

we have, for $T \rightarrow \infty$,

$$
z \simeq g_{3 / 2}(1)\left(\frac{T_{C}}{T}\right)^{3 / 2}
$$

Hence,

$$
\frac{1}{T_{2}}=\operatorname{Re} \phi(0) \longrightarrow 4 g_{3 / 2}(1)\left(\frac{S-x / 2}{g_{3 / 2}(1)}\right)^{2} \frac{F^{2}+G^{2}}{k_{B} T_{C}} \sqrt{\frac{T}{T_{C}}} .
$$

This is compatible with the experimental evidence [13] showing deviation (bending downward) from linear temperature dependence.

In all the experiments, $1 / T_{2}$ is a fraction of a Tesla. In view of equation (40), $F$ and $G$ being $1 \mathrm{~K}$ is enough to give the linewidth. A careful analysis of EPR data [29] indicated that this magnitude is reasonable. The only discrepancy between our calculation and experimental results is in the magnitude of the minimum $1 / T_{2}$. This discrepancy may be due to our treatment of the constraints. We have replaced the constraint on each site (equation (19)) by a global one (equation (36)). If the local constraints were restored, the correlation between spins became stronger. The fluctuation $\left(1 / T_{2}\right)$ would be suppressed. Improvement on this point is one of our ongoing projects.

It has been proposed in $[30,37,38]$ that linewidth has the following form over the hightemperature range:

$$
\Delta H_{p p}=\left[\chi_{0}(T) / \chi(T)\right] \Delta H_{p p}(T \rightarrow \infty),
$$

where $\chi(T)$ and $\chi_{0}(T)$ are, respectively, the susceptibilities of the spins with DE interaction and isolated spins. The argument is that, when the temperature is higher than all the energy scales, the response function $\langle[\dot{\mathbf{S}}(\tau), \dot{\mathbf{S}}]\rangle$ should be independent of temperature. Therefore it is interesting to examine whether double-exchange systems follow this relation. Equation (13) can be written in terms of Schwinger boson operators as follows:

$H=-\frac{1}{N(2 s+1)} \sum_{\mathbf{k}, \mathbf{p}, \mathbf{q}} E_{\mathbf{k}-\mathbf{p}-\mathbf{q}} f_{\mathbf{k}-\mathbf{q}}^{\dagger}\left(A_{\mathbf{p}} A_{\mathbf{p}+\mathbf{q}}^{\dagger}+B_{\mathbf{p}} B_{\mathbf{p}+\mathbf{q}}^{\dagger}\right) f_{\mathbf{k}}+\frac{g \mu_{\mathrm{B}} H_{0}}{2} \sum_{\mathbf{q}}\left(A_{\mathbf{q}}^{\dagger} A_{\mathbf{q}}-B_{\mathbf{q}}^{\dagger} B_{\mathbf{q}}\right)$.

The susceptibility has the form

$\chi(T)=-\frac{1}{V} \frac{\partial^{2} \mathcal{F}}{\partial H_{0}^{2}}=\frac{\beta}{V}\left(\frac{g \mu_{\mathrm{B}}}{2}\right)^{2}\left\{\left\langle\left[\sum_{\mathbf{q}}\left(A_{\mathbf{q}}^{\dagger} A_{\mathbf{q}}-B_{\mathbf{q}}^{\dagger} B_{\mathbf{q}}\right)\right]^{2}\right\rangle-\left\langle\sum_{\mathbf{q}}\left(A_{\mathbf{q}}^{\dagger} A_{\mathbf{q}}-B_{\mathbf{q}}^{\dagger} B_{\mathbf{q}}\right)\right\rangle^{2}\right\}$,

where $\mathcal{F}$ is the free energy. Note that

$$
\left\langle A_{\mathbf{q}}^{\dagger} A_{\mathbf{q}} A_{\mathbf{q}}^{\dagger} A_{\mathbf{q}}\right\rangle-\left\langle A_{\mathbf{q}}^{\dagger} A_{\mathbf{q}}\right\rangle^{2}=-\frac{1}{\beta} \frac{\partial n_{\mathbf{p}}}{\partial \omega_{\mathbf{p}}}
$$




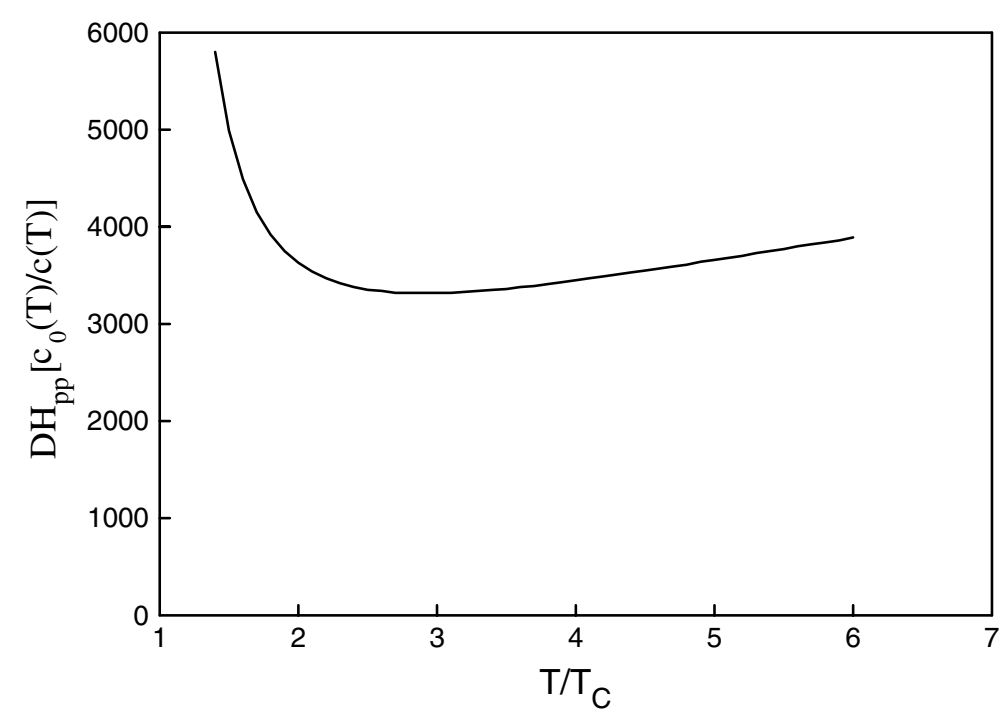

Figure 2. $\Delta H_{p p}\left[\chi(T) / \chi_{0}(T)\right]$ against $T / T_{C}$ for $F=G=2 \mathrm{~K}$, where $\chi(T)$ and $\chi_{0}(T)$ are the susceptibilities of the double-exchange system and isolated spins, respectively.

and

$$
\left\langle A_{\mathbf{q}}^{\dagger} A_{\mathbf{q}} B_{\mathbf{q}}^{\dagger} B_{\mathbf{q}}\right\rangle=\left\langle A_{\mathbf{q}}^{\dagger} A_{\mathbf{q}}\left(2 S-x-A_{\mathbf{q}}^{\dagger} A_{\mathbf{q}}\right)\right\rangle^{2} .
$$

As a result, we obtained

$$
\begin{aligned}
\chi(T) & =-\left(g \mu_{\mathrm{B}}\right)^{2} \sum_{\mathbf{q}} \frac{\partial n_{\mathbf{p}}}{\partial \omega_{\mathbf{p}}} \\
& =\frac{\left(g \mu_{\mathrm{B}}\right)^{2}}{8 \pi^{2} D} \sqrt{\frac{\pi k_{B} T}{D}} g_{1 / 2}(z),
\end{aligned}
$$

where $g_{1 / 2}(z)$ was defined in equation (38) and $z$, the fugacity, can be evaluated with equation (40). Thus we can plot $\Delta H_{p p}\left[\chi(T) / \chi_{0}(T)\right]$ against $T / T_{C}$. In figure 2 , we see that there is still reminiscent temperature dependence away from $T_{C}$, even though $\chi(T) / \chi_{0}(T)$ is a monotonically decreasing function of $T$. The reason, we think, is that the constraints (19) exist throughout the temperature range. Through equations (39) and (40), their presence affected the value of fugacity, which, in turn, affected the temperature dependence of $\Delta H_{p p}$.

Perhaps our most important result is that $1 / T_{2}$ is a universal function of $T / T_{C}$. That is to say, the linewidth multiplied by $T_{C}$ plotted against $T / T_{C}$ from different systems, lies on the same curve. The doping concentration $x$ affected $T_{2}$ only through $T_{C}$. In practice, it may be more complicated. SE interaction and Jahn-Teller distortion will affect $T_{C}$ and they are not accounted for by our calculation. However, there is experimental evidence for the universal behaviour. In [13], $\Delta H_{p p}(T) / \Delta H_{p p}(\infty)$ versus $T / T_{C}$ from various systems were plotted and they lie on a same line. In our calculation, we do not have a $\Delta H_{p p}(\infty)$. However, if we divide $\Delta H_{p p}(T)$ or $1 / T_{2}$ by a $\Delta H_{p p}$ at a sufficiently high temperature, the factor $\left(F^{2}+G^{2}\right) / k_{B} T_{C}$ will be cancelled and a universal curve will appear. 


\section{Acknowledgment}

This work was partially supported by the National Science Council (contract number NSC 92-2112-M-002-038).

\section{Appendix}

Here, we consider the contribution of the self-energy of boson propagators to the computation of equation (30). Applying the Matsubara formalism [40], we change the summation into integration:

$\frac{1}{\beta} \sum_{m, \mathbf{p}, \mathbf{q}} D\left(\mathrm{i} \omega_{m}, \mathbf{p}\right) D\left(\mathrm{i} \omega_{n}+\mathrm{i} \omega_{m}, \mathbf{q}\right)=-\frac{1}{2 \pi \mathrm{i}} \sum_{\mathbf{p}, \mathbf{q}} \int_{C} \mathrm{~d} z D(z, \mathbf{p}) D\left(z+\mathrm{i} \omega_{n}, \mathbf{q}\right) n(z)$,

where contour $C$ encloses all the poles except those of $n(z)$. Owing to the self-energy, the propagator has a continuous distribution of poles. They form a branch cut. The contour can now be deformed so that the integration paths are along each side of the cuts:

$$
\begin{aligned}
\frac{1}{\beta} \sum_{m, \mathbf{p}, \mathbf{q}} D\left(\mathrm{i} \omega_{m}, \mathbf{p}\right) & D\left(\mathrm{i} \omega_{n}+\mathrm{i} \omega_{m}, \mathbf{q}\right) \\
= & -\frac{1}{2 \pi \mathrm{i}} \sum_{\mathbf{p}, \mathbf{q}} \int \mathrm{d} z n(z)\left\{D(z, \mathbf{p})\left[D\left(z+\mathrm{i} \omega_{n}+\mathrm{i} \delta, \mathbf{q}\right)-D\left(z+\mathrm{i} \omega_{n}-\mathrm{i} \delta, \mathbf{q}\right)\right]\right. \\
& +D\left(z+\mathrm{i} \omega_{n}, \mathbf{q}\right)[D(z+\mathrm{i} \delta, \mathbf{p})-D(z-\mathrm{i} \delta, \mathbf{p})] .
\end{aligned}
$$

Using the properties of propagators, we have

$$
\begin{aligned}
\frac{1}{\beta} \sum_{m, \mathbf{p}, \mathbf{q}} D\left(\mathrm{i} \omega_{m}, \mathbf{p}\right) & D\left(\mathrm{i} \omega_{n}+\mathrm{i} \omega_{m}, \mathbf{q}\right) \\
= & -\frac{1}{2} \sum_{\mathbf{p}, \mathbf{q}} \int \mathrm{d} z n(z)\left[D\left(z-\mathrm{i} \omega_{n}, \mathbf{p}\right)+D\left(z+\mathrm{i} \omega_{n}, \mathbf{p}\right)\right] \operatorname{Im} \mathbf{D}(z, \mathbf{q}) .
\end{aligned}
$$

When computing $\operatorname{Re} \phi$ from $\operatorname{Im} \psi$, we need to take the derivative with respect to $\omega_{n}$ and then set $\omega_{n}$ to 0 (see equation (11)),

$$
\begin{aligned}
\frac{1}{\mathrm{i} \beta} \frac{\partial}{\partial \omega_{n}} \sum_{m, \mathbf{p}, \mathbf{q}} D & \left(\mathrm{i} \omega_{m}, \mathbf{p}\right) D\left(\mathrm{i} \omega_{n}+\mathrm{i} \omega_{m}, \mathbf{q}\right) \\
& =\frac{1}{2} \int \mathrm{d} z\left[D\left(z+\mathrm{i} \omega_{n}, \mathbf{p}\right)-D\left(z-\mathrm{i} \omega_{n}, \mathbf{p}\right)\right] \frac{\partial}{\partial z}[\operatorname{Im} \mathbf{D}(z, \mathbf{q}) n(z)],
\end{aligned}
$$

where integration by parts and interchanging of $\mathbf{p}$ and $\mathbf{q}$ were made. By setting $\omega_{n}$ equal to zero, the right hand side of equation (A.4) becomes

$$
\begin{array}{rl}
\mathrm{i} \pi \sum_{\mathbf{p}, \mathbf{q}} \int \mathrm{d} z \operatorname{Im} D & D(z, \mathbf{p}) \frac{\partial}{\partial z}[\operatorname{Im} \mathbf{D}(z, \mathbf{q}) n(z)] \\
& =\frac{\mathrm{i} \pi}{2} \sum_{\mathbf{p}, \mathbf{q}} \int \mathrm{d} z \operatorname{Im} D(z, \mathbf{p}) \operatorname{Im} \mathbf{D}(z, \mathbf{q}) \frac{\partial \mathbf{n}(\mathbf{z})}{\partial \mathbf{z}}
\end{array}
$$

and, thus, gives the factor $\partial n(z) / \partial z$. The vertex correction usually gives a multiplying factor and, hence, is unlikely to neutralize it. 


\section{References}

[1] Jonker G H and Santen J H 1950 Physics 16337

[2] Kusters R M et al 1989 Physica 155B 362

Jin S et al 1994 Science 264413

Tokura Y et al 1994 J. Phys. Soc. Japan 633931

[3] See e.g. Gagotto E, Hotta T and Moreo A 2001 Phys. Rep. 3441

[4] Zener C 1951 Phys. Rev. 82403

[5] Anderson P W and Hasegawa 1955 Phys. Rev. 100675

[6] de Gennes P G 1960 Phys. Rev. 118141

[7] Millis A J, Littlewood P B and Shraiman B I 1995 Phys. Rev. Lett. 745144

Millis J, Shraiman B I and Mueller R 1996 Phys. Rev. Lett. 77175

[8] See e.g. Mori S, Chen C H and Cheong S-M 1998 Nature 392473

[9] Shengelaya A et al 1996 Phys. Rev. Lett. 775296

[10] Oseroff B et al 1996 Phys. Rev. B 536521

[11] Lofloand S E et al 1997 Phys. Lett. A 233476

[12] Rettori C et al 1997 Phys. Rev. B 553083

[13] Causa M T et al 1998 Phys. Rev. B 583233

[14] Gundakaram R, Lin J G and Huang C Y 1998 Phys. Rev. B 586521

[15] Ivanshin V A et al 1999 Phys. Rev. B 616123

[16] Pake G E 1962 Paramagnetic Resonance (New York: Benjamin)

[17] Kubo R 1965 Prog. Phys. Rep. 29255

[18] Kawabata A 1970 J. Phys. Soc. Japan 29902

[19] Mori H 1965 Prog. Theor. Phys. 33423

[20] Ting C S and Nee T W 1986 Phys. Rev. B 337056

[21] Kubo K and Ohata N 1972 J. Phys. Soc. Japan 3321

[22] Khaliullin G and Kilian R 2000 Phys. Rev. B 613494

[23] Weisse A, Loos J and Fehske H 2001 Phys. Rev. B 64 054406-1

[24] Hu C D 2002 Phys. Rev. B 66132404

[25] See e.g. Auerbach A 1994 Interacting Electron and Quantum Magnetism (New York: Springer)

[26] Vaks V G, Larkin A I and Pikin S A 1969 JETP 26188

[27] Moriya T 1960 Phys. Rev. 12091

[28] Alejandro G, Passeggi M C G, Vega D, Ramos C A, Causa M T, Tovar M and Senis R 2003 Phys. Rev. B 68214429

[29] Deisenhofer J, Eremin M V, Zakharov D V, Ivanshin V A, Eremina R M, Krug von Nidda H-A, Mukhin A A, Balbashov A M and Loidl A 2002 Phys. Rev. B 65104440

[30] Huber D L, Alejandro G, Caneiro A, Causa M T, Prado F, Tovar M and Oseroff S B 1999 Phys. Rev. B 6012155

[31] Deisenhofer J, Kochelaev B I, Shilova E, Balbashov A M, Loidl A and Krug von Nidda H-A 2003 Phys. Rev. B 68214427

[32] Radaelli P G, Iannone G, Marezio M, Hwang H Y, Cheong S-W, Jorgensen J D and Argyrion D N 1997 Phys. Rev. B 568265

[33] Abragam A and Bleaney B 1970 Electron Paramagnetic Resonance of Transition Ions (Oxford: Clarendon Press)

[34] Hirsch J E and Tang S 1989 Phys. Rev. B 392850

[35] Huber D L and Seehra M S 1975 J. Phys. Chem. Solids 36723

[36] Dormann E and Jaccarino V 1974 Phys. Lett. 48A 81

[37] Huber D L 1971 J. Phys. Chem. Solids 322145

[38] Huber D L 1972 Phys. Rev. B 63180

[39] Pathria R K 1977 Statistical Mechanics (Oxford: Pergamon)

[40] Mahan G D 1981 Many-Particle Physics (New York: Plenum) 